\title{
SOME FIXED POINT THEOREMS FOR CONDENSING MULTIFUNCTIONS IN LOCALLY CONVEX SPACES
}

\author{
C. H. SU AND V. M. SEHGAL
}

\begin{abstract}
Let $G$ be a nonempty subset of a locally convex space $E$ such that $\mathrm{cl}(G)$ is convex and quasi-complete, and $f: \mathrm{cl}(G) \rightarrow E$ a continuous condensing multifunction. In this paper, several fixed point theorems are established if $f$ satisfies some conditions on the boundary of $G$. The results herein extend some theorems of Reich [9] and generalize some of the well-known fixed point theorems.
\end{abstract}

The classical Tychonoff's fixed point theorem [10] has been extended to multifunctions by Browder [3], Fan [4], Glicksberg [5], Himmelberg [7] and several others. In a recent paper [9], Reich has proved some interesting fixed point theorems for condensing multifunctions and has given extensions of some of the results contained in [3] and [4]. In this paper, we prove several fixed point theorems for condensing multifunctions in a locally convex space. The main result of this paper (Theorem 2) is motivated by Reich [9] and extends a result in [9]. The results herein also generalize some wellknown results (see [2], [8]).

1. Let $X$ and $Y$ be topological spaces. A multifunction $f: X \rightarrow Y$ is a point to set function such that for each $x \in X, f(x)$ is a nonempty subset of $Y$. The multifunction $f: X \rightarrow Y$ is (a) upper semicontinuous (u. s. c.) iff for each closed subset $B$ of $Y$, the set $f^{-1}(B)=\{x: f(x) \cap B \neq \varnothing\}$ is a closed subset of $X$, (b) lower semicontinuous (l.s.c.) iff for each open subset $U \subseteq Y$, the set $f^{-1}(U)$ is an open subset of $X,(\mathrm{c})$ continuous iff it is both u.s.c. and l.s.c., (d) point-compact (closed, convex) iff for each $x \in X, f(x)$ is a compact (closed, convex) subset of $Y$.

It follows immediately from the above that a multifunction $f: X \rightarrow Y$ is u.s.c. iff for each $x \in X$ and open $U \subseteq Y$ with $f(x) \subseteq U$, there is an open $V \subseteq X$ such that $x \in V$ and $f(V)=\bigcup\{f(z): z \in V\} \subseteq U$. It is 1.s.c. iff for each open set $U \subseteq Y$ and each $x \in X$ with $f(x) \cap U \neq \varnothing$, there exists an

Received by the editors February 16, 1974.

AMS (MOS) subject classifications (1970). Primary 47H10, 54H20.

Key words and phrases. Multifunction, condensing, fixed point, quasi-complete. 
open set $V \subseteq X$ such that $x \in V$ and $f(z) \cap U \neq \varnothing$ for each $z \in V$.

Proposition 1. Let $f: X \rightarrow Y$ be a u.s.c., point-compact multifunction and $\left\{x_{a}: a \in \Gamma\right\}$ a net in $X$ such that $x_{a} \rightarrow x_{0}$. If $y_{a} \in f\left(x_{\alpha}\right)$ for each $\alpha \in \Gamma$, then there is a $y_{0} \in f\left(x_{0}\right)$ and a subnet $\left\{y_{\beta}\right\}$ of the net $\left\{y_{a}: \alpha \in \Gamma\right\}$ such that $y_{\beta} \rightarrow y_{0}$.

Proof. Suppose that no subnet of the net $\left\{y_{\alpha}: \alpha \in \Gamma\right\}$ converges to a point in $f\left(x_{0}\right)$. Then for each $y \in f\left(x_{0}\right)$, there is an open neighborhood $U(y)$ of $y$ such that $y_{a} \notin U(y)$ eventually. Since $f\left(x_{0}\right)$ is compact, there is a finite subset $\left\{y_{i}: i=1,2, \ldots, n\right\} \subseteq f\left(x_{0}\right)$ such that

$$
f\left(x_{0}\right) \subseteq \bigcup\left\{U\left(y_{i}\right): i=1,2, \ldots, n\right\}=U
$$

and $y_{a} \notin U$ eventually. Now, $f$ being u.s.c., it follows from (1) that there is open neighborhood $V$ of $x_{0}$ such that $f(V) \subseteq U$. However, this implies that $f\left(x_{\alpha}\right) \subseteq U$ eventually and hence, $y_{\alpha} \in U$ eventually, a contradiction.

2. Throughout this section let $E$ denote a locally convex separated topological vector space and $\mathcal{U}$ a base of absolutely convex neighborhoods of the origin $\theta$. A subset $X$ of $E$ is totally bounded iff for each $U \in \mathcal{U}$, there exists a finite subset $B \subseteq X$ such that $X \subseteq B+U$. For any subset $A$ of $E$, let

$Q(A)=\{U \in \mathcal{U}: A \subseteq B+U$ for some totally bounded subset $B$ of $E\}$. Let $S \subseteq E$. A multifunction $f: S \rightarrow E$ is condensing iff $Q(A) \varsubsetneqq Q(f(A))$ for each bounded but not totally bounded subset $A \subseteq S$ (see Himmelberg, Porter, Van Vleck [6]).

For a subset $S \subseteq E$, let $\partial S$ denote the boundary of $S$ in $E$, co $(S)$ the convex hull of $S$. A closed subset $B$ of $E$ is quasi-complete if its closed bounded subsets are complete. It is clear that if $X$ is a totally bounded subset of a quasi-complete subset $B$ then $\mathrm{cl}(X)$ is compact.

The following lemma is similar to Theorem 1 in [6] and the proof therein works verbatim with the hypothesis of this lemma.

Lemma 1. Let $X$ be a quasi-complete convex subset of $E$ and $f: X \rightarrow X$ be an u.s.c., point-compact, point-convex, condensing multifunction. If $f(X)$ is bounded, then $f$ has a fixed point in $X$, that is, there is an $x_{0} \in X$ such that $x_{0} \in f\left(x_{0}\right)$.

Theorem 1. Let $X$ be a convex, quasi-complete subset of $E$, and $f:$ $X \rightarrow E$ an u.s.c., point-compact, point-convex, condensing multifunction. If $f(X)$ is bounded and $f(x) \cap X \neq \varnothing$ for each $x \in X$, then $f$ has a fixed point in $X$. 
Proof. Let $F(x)=f(x) \cap X$. Then $F$ satisfies the conditions of Lemma 1 , and hence there is an $x_{0} \in X$ such that $x_{0} \in F\left(x_{0}\right) \subseteq f\left(x_{0}\right)$.

The following consequences of Theorem 1 are immediate.

Corollary 1. Let $X$ be a convex, quasi-complete subset of $E$ and $f$ : $X \rightarrow E$ be an u.s.c., point-compact, point-convex, condensing multifunction. If there exists a bounded subset $K$ of $X$ such that $f(x) \cap K \neq \varnothing$ for all $x \in X$, then $f$ has a fixed point in $X$.

Corollary $\dot{2}$. Let $X$ be a convex, quasi-complete subset of $E$ and $K a$ totally bounded subset of $X$. If $f: X \rightarrow E$ is an u.s.c., point-closed, pointconvex multifunction such that $f(x) \cap K \neq \varnothing$ for all $x \in X$, then $f$ has a fixed point in $X$.

Since for any subset $G \subseteq E, \partial(\operatorname{cl}(G)) \subseteq \partial(G)$, the following result extends a recent result of Reich [9, Theorem 3.5].

Theorem 2. Let $G$ be a nonempty open subset of $E$ such that $\mathrm{cl}(G)$ is convex and quasi-complete. If $f: \mathrm{cl}(G) \rightarrow E$ is a continuous, point-compact, point-convex, condensing multifunction satisfying the conditions (a) $f(G)$ is bounded, (b) there is a $w \in G$ such that for all $y \in \partial(\mathrm{cl}(G))$ and $z \in f(y)$, $z-w=m(y-w)$ implies $m \ngtr 1$, then $f$ has a fixed point in $\mathrm{cl}(G)$.

Proof. Let $p$ be the Minkowski functional of the set cl: $(G)-w$. Define a mapping $q: \operatorname{cl}(G) \rightarrow(0,1]$ by

$$
q(x)=[\max \{1, \min \{p(y-w): y \in f(x)\}\}]^{-1} .
$$

Note that since $f(x)$ is compact and $p$ is continuous, the minimum in (3) exists at some element of $f(x)$. Define a multifunction $g: \mathrm{cl}(G) \rightarrow E$ by

$$
g(x)=q(x) f(x)+(1-q(x)) w .
$$

Note that for each $x \in \mathrm{cl}(G)$, there is a $y \in f(x)$ such that $p(y-w)=$ $\min \{p(z-w): z \in f(x)\}$ and hence $z=q(x) y+(1-q(x)) w \in g(x)$. Moreover, for this $y$, it follows from (3) that $p(z-w)=q(x) p(y-w) \leq 1$. Therefore $z \in \mathrm{cl}(G)$ and hence $\operatorname{cl}(G) \cap g(x) \neq \varnothing$ for any $x \in \mathrm{cl}(G)$. We show that $g$ satisfies the conditions of Theorem 1. It is clear from (4) that $g(x)$ is compact and convex for each $x$. Further, since $g(\operatorname{cl}(G)) \subseteq \operatorname{co}(f(\operatorname{cl}(G)) \cup\{w\})$, it follows by hypothesis (a) that $g(\mathrm{cl}(G))$ is bounded. Now, for any bounded but not totally bounded subset $A$,

$$
Q(g(A)) \supseteq Q(\operatorname{co}(f(A) \cup\{w\}))=Q(f(A)) \supsetneqq Q(A) .
$$

It follows from (5) that $g$ is condensing. We show that $g$ is u.s.c. Let $B$ 
be a closed subset of $E$ and let $A=g^{-1}(B)$. We show that $A$ is closed. Let $x_{0} \in \mathrm{cl}(A)$ and let a net $\left\{x_{a}: \alpha \in \Gamma\right\}$ in $A$ be convergent to $x_{0}$. For each $a \in \Gamma$, choose $y_{\alpha} \in f\left(x_{\alpha}\right)$ and a $y_{0} \in f\left(x_{0}\right)$ such that $p\left(y_{a}-w\right)=$ $\min \left\{p(y-w): y \in f\left(x_{a}\right)\right\}$ and $p\left(y_{0}-w\right)=\min \left\{p(y-w): y \in f\left(x_{0}\right)\right\}$. It follows by Proposition 1 that there is a subnet $\left\{y_{\beta}: \beta \in \Gamma_{1}\right\}$ of the net $\left\{y_{\alpha}: \alpha \in \Gamma\right\}$ converging to a $y \in f\left(x_{0}\right)$. Now, since $p$ is continuous, for any $\epsilon>0$, there is $\beta_{1} \in \Gamma_{1}$ such that for all $\beta \geq \beta_{1}$

$$
p\left(y_{0}-w\right) \leq p(y-w) \leq p\left(y_{\beta}-w\right)+\epsilon \text {. }
$$

Let $U=\left\{z \in E: p(z-w)<p\left(y_{0}-w\right)+\epsilon\right\}$. Then $U$ is open and $y_{0} \epsilon$ $U \cap f\left(x_{0}\right)$. Now, $f$ being l.s.c., there is a neighborhood $V$ of $x_{0}$ such that $f(z) \cap U \neq \varnothing$ for each $z \in V$ and hence there is a $\beta_{2} \in \Gamma_{1}$ such that $f\left(x_{\beta}\right) \cap U \neq \varnothing$ for all $\beta \geq \beta_{2}$. This implies that for $\beta \geq \beta_{2}$

$$
p\left(y_{\beta}-w\right)<p\left(y_{0}-w\right)+\epsilon \text {. }
$$

It follows now from (6) and (7) that for all $\beta \geq \max \left\{\beta_{1}, \beta_{2}\right\}$,

$$
p\left(y_{0}-w\right) \leq p(y-w) \leq p\left(y_{\beta}-w\right)+\epsilon<p\left(y_{0}-w\right)+2 \epsilon .
$$

Since $\epsilon>0$ is arbitrary, we have from (8) that $p\left(y_{0}-w\right)=p(y-w)$ and $q\left(x_{\beta}\right) \rightarrow q\left(x_{0}\right)$. Since for any $\beta \in \Gamma_{1}, g\left(x_{\beta}\right) \cap B \neq \varnothing$, there is $z_{\beta} \in f\left(x_{\beta}\right)$ such that

$$
q\left(x_{\beta}\right) z_{\beta}+\left(1-q\left(x_{\beta}\right)\right) w \in B .
$$

By Proposition 1 , there is a subnet $\left\{z_{\delta}\right\}$ of the net $\left\{z_{\beta}\right\}$ and a $z_{0} \in f\left(x_{0}\right)$ such that $z_{\delta} \rightarrow z_{0}$. Since $q\left(x_{\delta}\right) \rightarrow q\left(x_{0}\right)$, therefore, from (9) it follows that $g\left(x_{0}\right) \cap B \neq \varnothing$, that is $x_{0} \in A$. Thus, $g$ is an u.s.c. multifunction, and satisfies the conditions of Theorem 1 , and hence there is $u \in \mathrm{cl}(G)$ with $u \in$ $g(u)$. We assert that $\alpha=\min \{p(y-w): y \in f(u)\} \leq 1$. Suppose $\alpha>1$. Let $u=q(u) y_{1}+(1-q(u)) w \in g(u)$ for some $y_{1} \in f(u)$. By our assumption $p\left(y_{1}-w\right)>1$, and hence by the definition of $q$, it follows that $p(u-w)=$ $q(u) p\left(y_{1}-w\right) \geq 1$. Since $u \in \mathrm{cl}(G)$, the last inequality implies that $u \in$ $\lambda(\mathrm{cl}(G))$. Also $y_{1}-w=[q(u)]^{-1}(u-w),[q(u)]^{-1}>1$. This contradicts hypothesis (b). Thus $a \leq 1$ and hence $q(u)=1$. This implies that $u \in$ $g(u)=f(u)$. This establishes the proof of Theorem 2 .

Corollary 3. Let $G$ be a nonempty open subset of $E$ such that $\mathrm{cl}(G)$ is convex and quasi-complete. Suppose $f: \mathrm{cl}(G) \rightarrow E$ is a continuous, pointcompact, point-convex, condensing multifunction such that (i) $f(G)$ is bounded, and (ii) $f(x) \subseteq \mathrm{cl}(G)$ for all $x \in \partial(\mathrm{cl}(G))$. Then $f$ has a fixed point in $\mathrm{cl}(G)$. 
Theorem 3. Let $G$ be a nonempty open subset of $E$ with $\mathrm{cl}(G)$ convex. Let $f: \mathrm{cl}(G) \rightarrow E$ be a continuous, point-closed, point-convex multifunction satisfying the conditions (i) $\mathrm{cl}(f(G))$ is compact, and (ii) there is a $w \in G$ such that for each $y \in \partial(\mathrm{cl}(G))$ and $z \in f(y), z-w=m(y-w)$ implies $m \ngtr 1$, then $f$ has a fixed point in $\mathrm{cl}(G)$.

Proof. Define the functions $q$ and $g$ as in Theorem 2. Then $g$ is u.s.c. and $g(x) \cap \operatorname{cl}(G) \neq \varnothing$ for any $x \in \mathrm{cl}(G)$. Since

$$
g(\mathrm{cl}(G)) \subseteq[0,1] \mathrm{cl}(f(G))+[0,1] w
$$

and the right side of (10) is compact, therefore $\mathrm{cl}(\mathrm{g}(\mathrm{cl}(G)))$ is compact. Define a multifunction $h: \mathrm{cl}(G) \rightarrow \mathrm{cl}(G)$ by $h(x)=g(x) \cap \mathrm{cl}(G)$. Then $h$ is u.s.c. and $h(\mathrm{cl}(G)) \subseteq g(\mathrm{cl}(G))$. Thus, by Himmelberg's theorem [7, Theorem 2], there is a $u \in \mathrm{cl}(G)$ such that $u \in h(u)$. It can now be shown, as in Theorem 2 , that $u \in h(u) \subseteq g(u)=f(u)$.

The following consequence of Theorem 3 is an extension of a recent result of Porter [8] and generalizes a result of Singball [2].

Corollary 4. Let $G$ be a nonempty open subset of $E$ such that $\mathrm{cl}(G)$ is convex. If $f: \mathrm{cl}(G) \rightarrow E$ is a continuous, point-closed and point-convex multifunction such that $\mathrm{cl}(f(G))$ is compact and $f(\partial(\mathrm{cl}(G))) \subseteq G$, then $f$ has a fixed point in $\mathrm{cl}(G)$.

\section{REFERENCES}

1. C. Berge, Topological spaces, Macmillan, New York, 1963.

2. F. F. Bonsall, Lectures on some fixed point theorems of functional analysis, Tata Institute of Fundamental Research, Bombay, 1962. MR 33 \#6332.

3. F. E. Browder, The fixed point theory of multi-valued mappings in topological vector spaces, Math. Ann. 177 (1968), 283-301. MR 37 \# 4679.

4. K. Fan, Fixed-point and minimax theorems in locally convex topological linear spaces, Proc. Nat. Acad. Sci. U. S. A. 38 (1952), 121-126. MR 13, 858.

5. I. L. Glicksberg, A further generalization of Kakutani fixed point theorem, with application to Nash equilibrium points, Proc. Amer. Math. Soc. 3 (1952), 170-174. MR 13, 764 .

6. C. J. Himmelberg, J. R. Porter and F. S. Van Vleck, Fixed point theorems for condensing multifunctions, Proc. Amer. Math. Soc. 23 (1969), 635-641. MR 39 \# 7480 .

7. C. J. Himmelberg, Fixed points of compact multifunctions, J. Math. Anal. Appl. 38 (1972), 205-207. MR 46 \# 2505.

8. A. J. B. Porter, An elementary version of the Leray-Schauder theorem, J. Lond on Math. Soc. (2) 5 (1972), 414-416.

9. S. Reich, Fixed points in locally convex spaces, Math. Z. 125 (1972), 17-31. MR 46 \# 6110 .

10. A. Tychonoff, Ein Fixpunktsatz, Math. Ann. 111 (1935), 767-776. 